Proc. Estonian Acad. Sci. Eng., 2004, 10, 3, 185-197

\title{
Improvement of lock-in signal processing for applications in measurement of electrical bioimpedance
}

Toomas Parve and Raul Land

Department of Electronics, Tallinn University of Technology, Ehitajate tee 5, 19086 Tallinn, Estonia; parveto@edu.ttu.ee

Received 6 April 2004, in revised form 17 June 2004

\begin{abstract}
Improvement of the switching mode lock-in signal processing, based on narrowing of the pulse of the half wave of the rectangular form signal, is discussed. The solution is aimed to the diminishing of the phasor errors caused by the sensitivity of the switching mode lock-in signal converters to the odd higher harmonics of the input signal. This improvement is technically realizable without significant increasing of circuit complexity and energy consumption. The method is foreseen for electrical bioimpedance measurement units used in implantable and portable medical devices for making medical measurements in vivo.
\end{abstract}

Key words: lock-in signal processing, measurement errors, electrical bioimpedance, implantable medical devices.

\section{INTRODUCTION}

Electrical impedance $\dot{Z}$ of biological objects (Fig. 1a) or electrical bioimpedance (EBI) is measured with the aim to get information about the biological processes taking place inside the living organism $\left[{ }^{1}\right]$.

From the general measurement point of view $\left[{ }^{2}\right]$, there is no significant difference between the bioimpedance and other impedances, like the impedance of chemical cells, etc. But as biological objects are structurally complicated, the bio-impedance has also a complicated equivalent circuit. Generally at least the three-element equivalent circuit is used (Fig. 1b).

Still it is possible to measure directly only the active and reactive components $R$ and $X$ of the complex impedance $\dot{Z}=R+\mathrm{j} X$ (or $G$ and $B$ of the complex admittance 

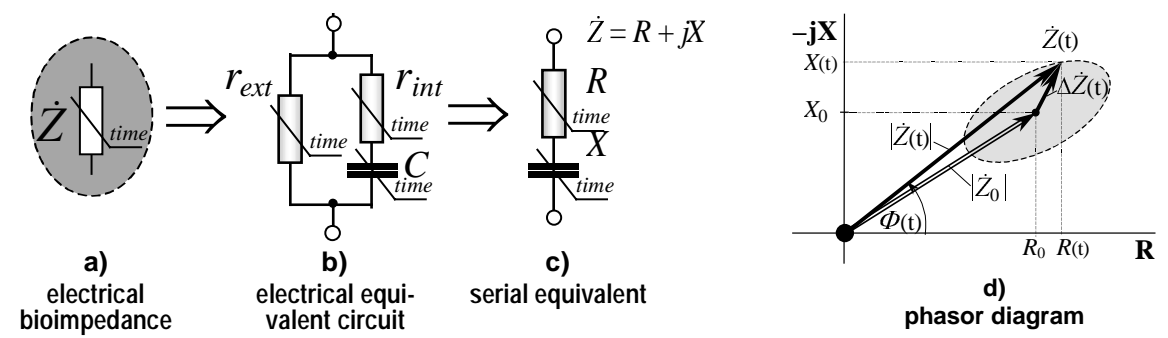

Fig. 1. Schematic presentation of electrical bioimpedance $\dot{Z}$ of a biological object (a), its electrical equivalents (b and c), and phasor diagram (d).

$Y=G+\mathrm{j} B$ ), which are mutually in quadrature (Fig. 1, c and d). To be used for mathematical conversions, $R$ and $X$ must be measured with required accuracy.

There are yet other specific features of the bioimpedance measurement.

\section{ASPECTS OF BIOIMPEDANCE APPLICATIONS}

In clinical environment, advanced measurement devices are used and correct measurement environment is created for bioimpedance measurement. Often fourterminal measurement (the Kelvin connection) is used to avoid effects from electrodes $\left[{ }^{3}\right]$.

Portable and especially implantable devices have to meet several restrictions from the side of energy consumption, size, weight, and also measurement techniques used. Of course, also the tasks, which are solved using the results of EBI measurement, are limited. Thus even simple measurement methods can give the results needed. For example, in the implantable cardiac pacemakers the EBI is often measured through the time response to a short pulse [ $\left.{ }^{1}\right]$. But mostly thanks to the fact that in the implantable devices the situation, in which the impedance is measured, is quite well fixed and stable in time. Thus very often the results of analysis of the response signal, achieved by using rectangular pulse waveform excitation signals (Fig. 2), are satisfactory.

Still it is rather difficult to interpret these results in accordance with the definition of impedance. Equivalent circuit of the bioimpedance is rather complicated, and the simple three-element equivalent is valid a few decades only. If we use sine wave excitation, then the frequency of the excitation signal can be selected in accordance with the validity range of the equivalent circuit. For the case of using the pulse form signals it is much harder to fulfil the corresponding demands to the excitation pulses. From the time response it is quite difficult to determine all the parameters with needed precision, as the effect of the main pole of the impedance function is strongly prevalent over the others, which have a small effect.

Yet more serious problems can rise from the electrical linearity of the biological object (organ, tissue). Low level of the measurement pulse leads to lower response and hence to higher uncertainties in measurement results. 


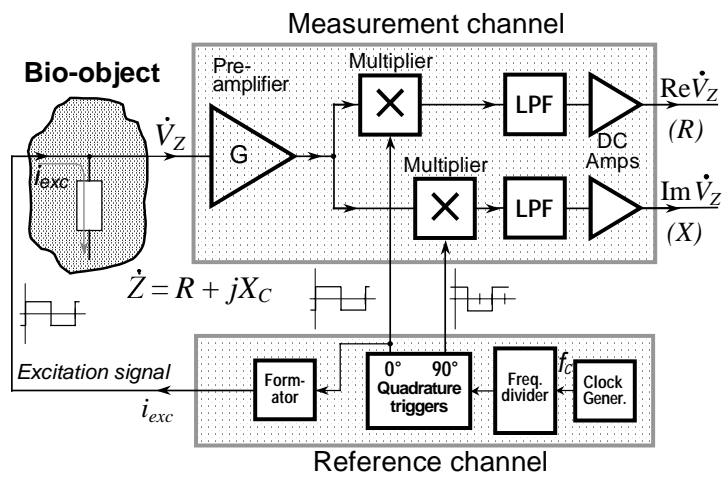

Fig. 2. Block diagram of lock-in bioimpedance analyser using rectangular waveforms.

Moreover, in the case of measurement of a living organism, the measurement situation is not static and is not exactly fixed. Often the measurement process is also affected by some disturbing signal of significant level, like the electrocardiographic (ECG) signal in the case of in vivo measurements of cardiovascular or lung impedance $\left[{ }^{1}\right]$. Then the simplest methods of measurement will not give proper results even if potentially very noise-proof methods, like the lock-in signal conversion, is used $\left[{ }^{2}\right]$.

It means that the accuracy of separation of the complex components $R$ and $X$ is important. The calibration accuracy is mostly given for certain phasor parameters $(R, X$, magnitude $Z$ or phase shift $\Phi$, Fig. $1 \mathrm{~d})$. In addition, not only the mean values of the parameters, but also their relatively small time variations, which are caused by biological processes in biological objects, are of interest. In some cases namely these small changes of the phasor parameters can be used as a basis for making medical decisions.

The situation is often quite complicated for the implantable devices, which must meet numerous other limitations concerning their size, power consumption, etc.

Although one can think about freely selecting the principle of impedance measurement, the lock-in signal conversion or synchronous detection (SD) method $\left[{ }^{2,3}\right]$ is most often chosen because of its inherent noise suppression ability and suitability for phase-sensitive measurements.

\section{LOCK-IN MEASUREMENT OF BIOIMPEDANCE}

\subsection{General considerations}

In the two channel lock-in system separate synchronous detectors (multipliers) of the in-phase and quadrature components allow to obtain the real and imaginary parts $R$ and $X$ of the complex impedance $\dot{Z}$ separately (Fig. 3). 


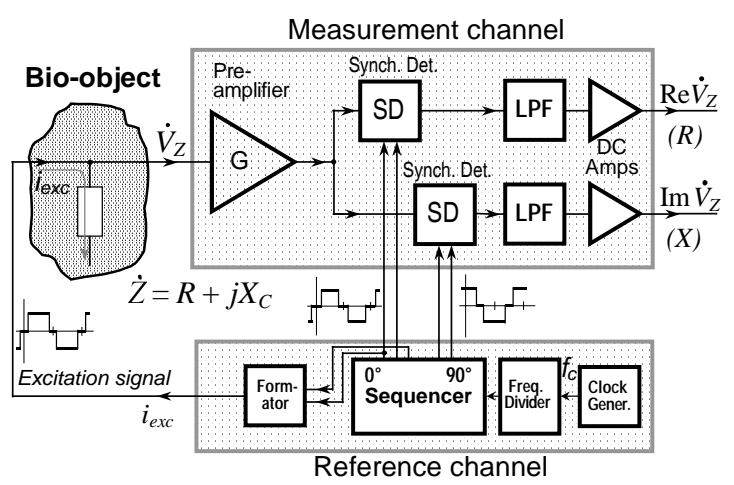

Fig. 3. Block diagram of lock-in bioimpedance analyser wherein modified rectangular waveforms are used.

As the complex impedance $\dot{Z}$ is defined for the case of the sine wave voltage and current, the excitation signal used for the measurement has also to be a sine wave. In the case of a linear circuit the response is also a sine wave. The EBI can be linearized only up to a certain level of the excitation signal.

Unfortunately, the synchronous or lock-in detection of the simple switching mode, known also as synchronous rectification, is strongly sensitive to the higher odd harmonics of the fundamental frequency of the excitation signal, depending on the content of harmonics in the rectangular reference waveform $\left[{ }^{2,4}\right]$. So the pure sine wave excitation signal should be used in synchronous rectifiers, and non-linearity of the object under investigation should not cause significant higher harmonics in the response signal $V_{Z}$.

As it is cumbersome to obtain the stable sine wave excitation in practical circuits, the rectangular waveform substitutes are commonly used in implantable units $\left[{ }^{1}\right]$. Correspondingly, also more complicated methods of synchronous signal conversion are to be used in electrical bioimpedance measurement.

\subsection{Modified lock-in measurement}

The aim is to modify the traditional lock-in system, operating with rectangular reference signals $\left[^{3}\right]$, without introducing significant complexity. What is needed is to reduce the higher odd harmonics content of the excitation signal and to decrease the sensitivity of the switching-type synchronous detectors to the lower order higher harmonics of the excitation signal using rectangular waves $\left[^{4-8}\right]$.

The simplest suitable approximation of the sine wave is obtained by narrowing the rectangular signal pulses and introducing the zero-level intervals (Fig. 4). That is, one can use three signal levels $(+\mathrm{A},-\mathrm{A}$ and 0$)$ instead of two (+A and $-\mathrm{A})$ as usual. This approach can be considered as an application of a specific pulse-width modulation to the pulse signal $\left[{ }^{9,10}\right]$. 


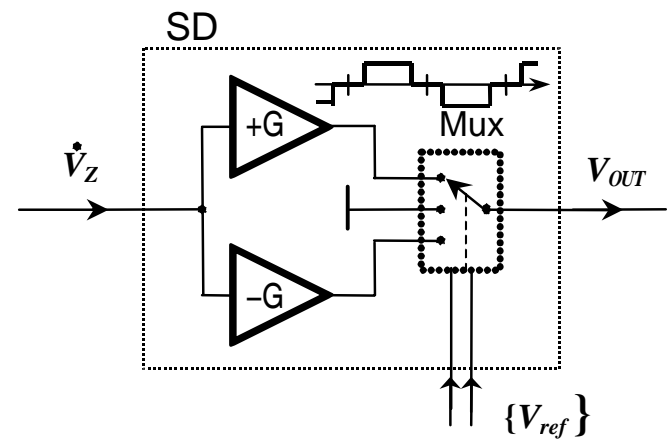

Fig. 4. Circuit diagram of the synchronous detector (SD) with narrowed pulse.

\subsection{Modified synchronous detector}

In Fig. 4, a modified synchronous detector is shown. Here only the multiplexer is modified in comparison with the ordinary switching type SD [ $\left.{ }^{2,3}\right]$. The third state of the multiplexer for obtaining zero gain (or zero signal level, or grounded output) is introduced to achieve the operating mode with narrowed pulse $\left[{ }^{9}\right]$.

Some changes must be introduced also in the generator of excitation and in reference signals. Fortunately, this is a trigger circuit, which is relatively easy to modify adding needed number of triggers $\left[{ }^{\circ}\right]$. Simultaneous $18^{\circ}$ and $30^{\circ}$ narrowing requires a clock frequency which is 60 times higher than the signals have $\left(f_{\mathrm{c}}=60 f\right)$. This is 15 times more than it is needed to get quadrature full cycle outputs $\left(f_{\mathrm{c}}=4 f\right)$. Of course, this also leads to the corresponding growth in the current consumption of the trigger circuit.

\subsection{Suppression of errors caused by harmonics}

A study of the spectral properties of the rectangular waveforms with narrowed pulse showed that from all the easy-to-generate waveform pairs with maximally different harmonic content, the best is the one consisting of waveforms having $18^{\circ}$ and $30^{\circ}$ pulse narrowing (Fig. 5b) $\left[{ }^{9}\right]$.

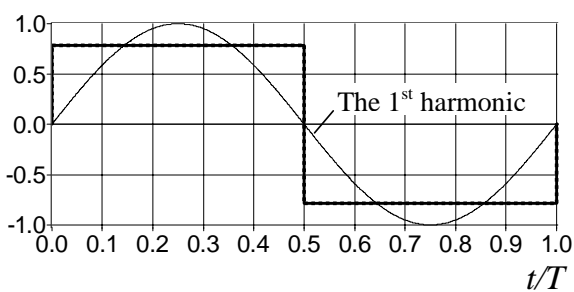

(a)

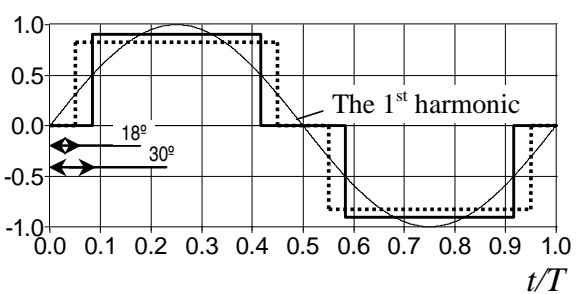

(b)

Fig. 5. Rectangular waveform reference and excitation signals (the case of zero phase-shift): (a) rectangular waveforms with a full duty cycle; (b) rectangular waveforms having duty cycle narrowed by $18^{\circ}$ and $30^{\circ}$ (the PWM). 
Outputs of the SD-s of in-phase and quadrature components in the case of rectangular waveform reference and excitation signals with full duty cycle (a) and with narrowed duty cycle (b), are shown in Fig. 6 for the full phase shift range $\Phi=-180^{\circ}$ to $+180^{\circ}$.

From the nearest higher odd harmonics there are not present the 3rd, 5th, and 9th harmonics at least in one of the waveforms. But the 7th harmonic exists in both of them though it is slightly suppressed in one of the signals (Fig. 7).

Using of the proposed narrowed rectangular waveforms gives significantly different phase sensitivity characteristics of the SD compared to those of the full duty cycle waveforms (Fig. 6). The results are very similar to those of the pure sine wave case, where the SD gain has a cosine form for the inphase SD output and the sine form for the quadrature SD output over the full phase cycle (from $-180^{\circ}$ to $+180^{\circ}$ in Fig. 5).

Comparison of phasor errors in the described two cases of using rectangular waveforms shows that the narrowed waveforms have a clear advantage over the

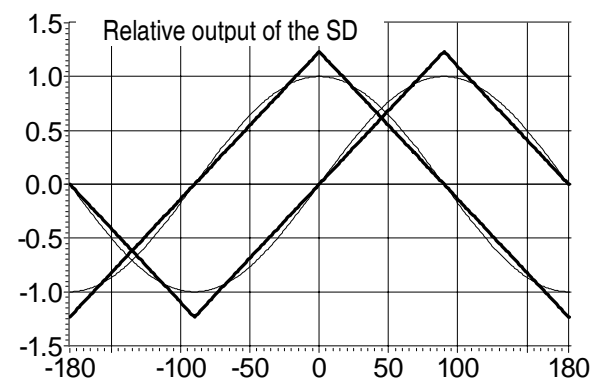

(a)

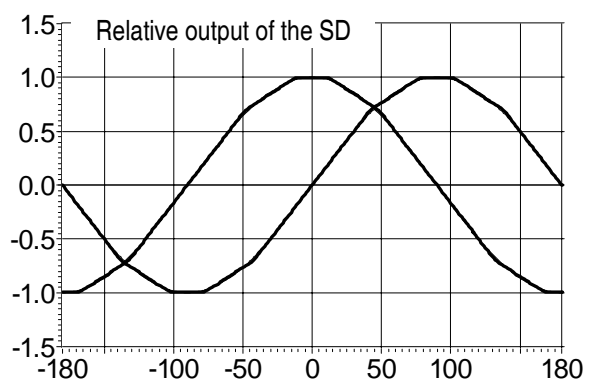

(b)

Fig. 6. In-phase and quadrature outputs in the case of rectangular waveform reference and excitation signals with full (a) and narrowed (b) duty cycles, shown in Fig. 5, for the phase shift from $-180^{\circ}$ to $+180^{\circ}$.

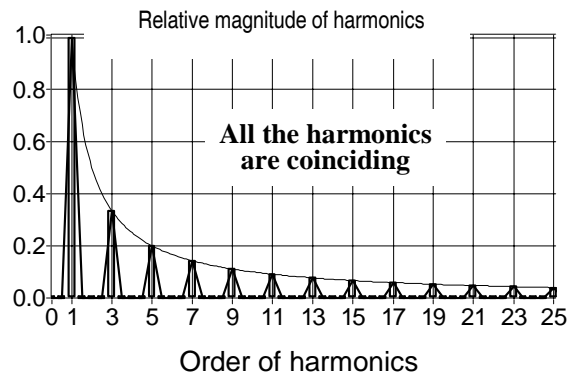

(a)

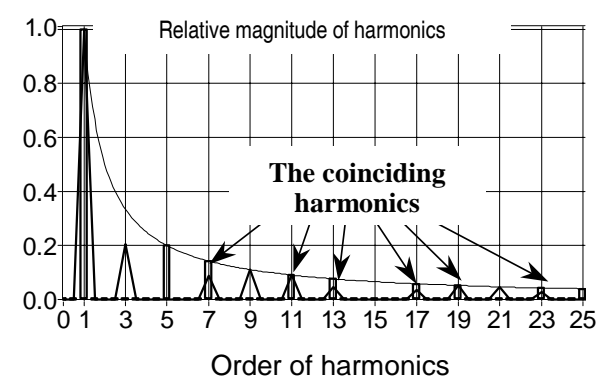

(b)

Fig. 7. Spectra of the rectangular waveform reference and excitation signals in the case of full duty cycle (a) and narrowed by $18^{\circ}$ and $30^{\circ}$ duty cycle (b). 


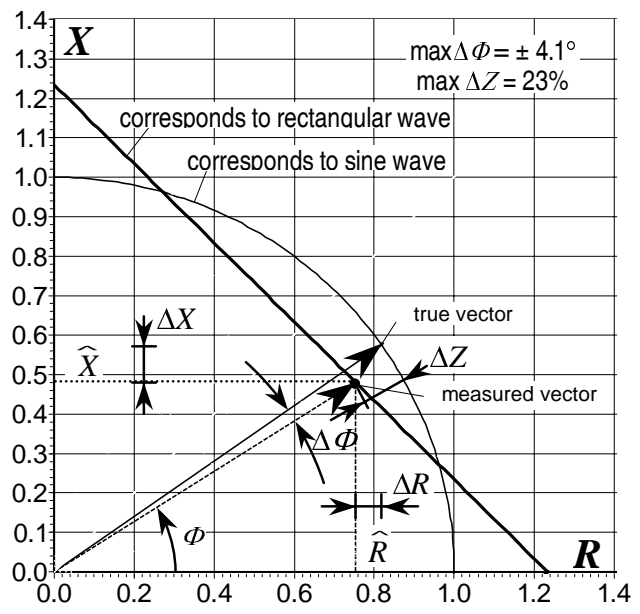

(a)

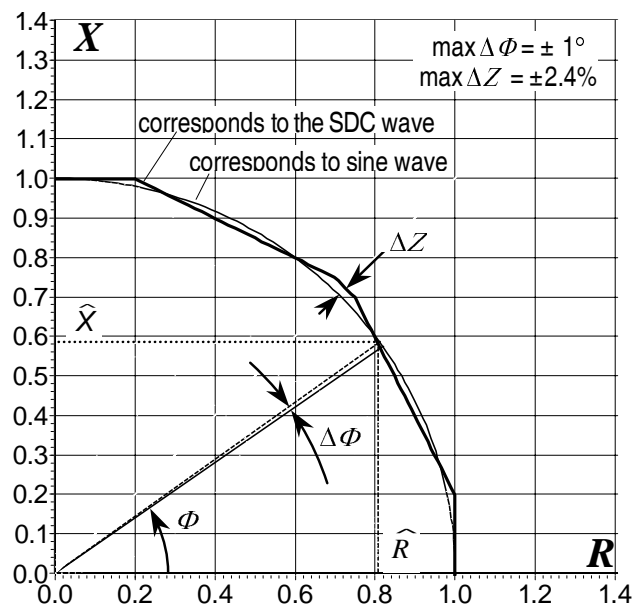

(b)

Fig. 8. Phasor errors (magnitude error $\Delta Z$ and phase error $\Delta \Phi$ ) due to application of rectangular waveforms: (a) the case of rectangular waveforms with full duty cycle; (b) the case of rectangular waveforms having narrowed by $18^{\circ}$ and $30^{\circ}$ duty cycle (SDC).
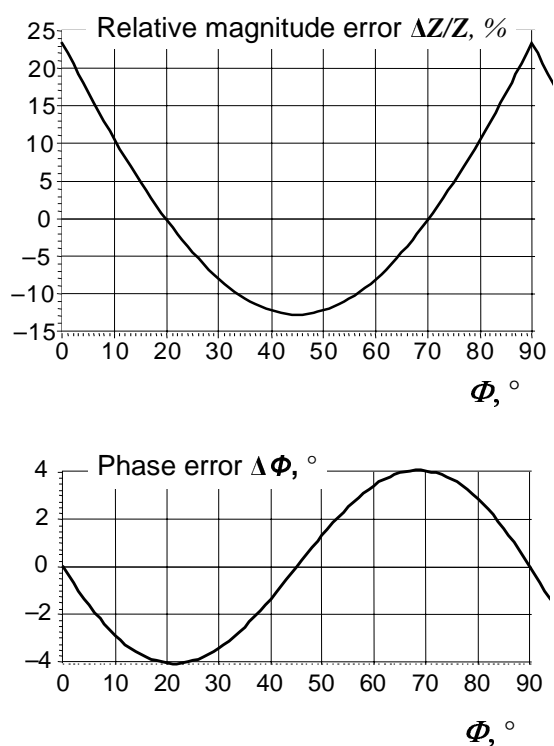

(a)
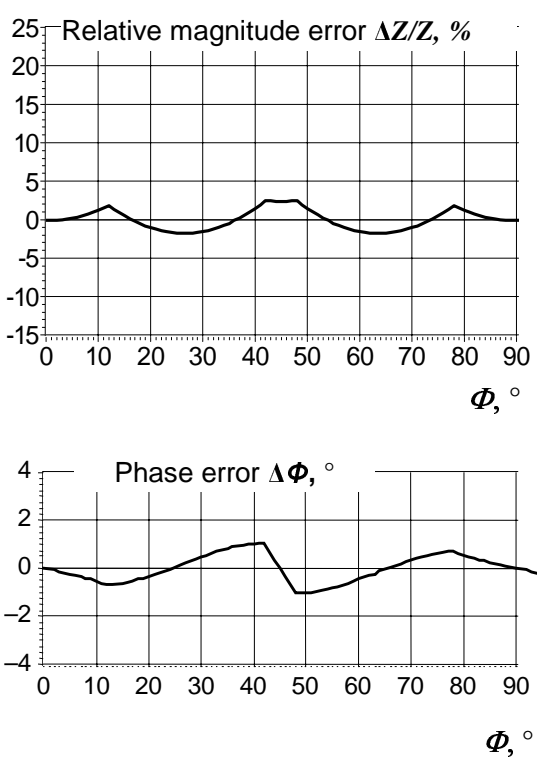

(b)

Fig. 9. Magnitude error $(\Delta Z)$ and phase error $(\Delta \Phi)$ in the case of using rectangular waveform signals instead of the sine-wave signals: (a) the case of rectangular waveforms with full duty cycle; (b) the case of rectangular waveforms having duty cycle narrowed by $18^{\circ}$ and $30^{\circ}$.

full duty cycle waveform (Fig. $8 \mathrm{a}$ and b). The ideal sine wave input gives us the perfect arc of circle as the trajectory of phasor tip over the first phase quadrant 
(fine line in Fig. 8, a and b). This trajectory can be considered as a basis for comparison.

The in-phase and quadrature outputs of the full duty cycle SD draw the straight sloped line, which is a side of a rectangle in the case of full duty cycle excitation waveform. The straight line is quite different from the arc of circle obtained in the ideal sine wave case, and corresponding magnitude and phase errors (Fig. 8a) can be evidently very large.

In the case of narrowed pulse waveforms, the SD outputs fit to the ideal sine wave case quite well, though the waveforms in fact remain rectangular. The corresponding magnitude errors are about ten and phase errors about four times smaller than in the case of the full duty cycle (Fig. 9).

\subsection{The frequency response measurement}

The frequency response of bioimpedance $\dot{Z}$ has capacitive character in principle, which means falling of the magnitude $Z$ of impedance towards higher frequencies $\left[{ }^{3}\right]$. The slope of the frequency response is not steep due to the presence of several dielectric dispersions in the biological objects $\left[^{3}\right]$. Though the bioimpedance $Z$ can not have a rise at the frequencies of higher harmonics, the same can not be said about the imaginary part $X$ of the impedance $\dot{Z}$.

As a result, no higher harmonic in the response voltage $V_{Z}$ can become relatively bigger than it is in the excitation current. Therefore, we can consider the harmonic content of the excitation signal as the worst case for the response signal. Evidently, the situation in the case of measurement of admittance is worse.

To perform the frequency response analysis of the bioimpedance, one has to vary the frequency or to apply the excitation at least at two frequencies, which are sufficiently different.

If the frequency response measurements is performed using the sine wave test signal and the non-linearity is negligible, no systematic error from processing of excitation and reference signals should appear independent from the frequency response of the complex impedance $\dot{Z}$ or its components.

But if we use a simpler solution with rectangular waveforms or other stepwise approximations of the sine wave, systematic errors of the lock-in signal conversion from the responses appear. The question is, how big can be errors of this kind in the case of bioimpedance measurement.

To get an imagination, the three-element equivalent circuit (Fig. 1b) was taken as the reference with the values of the parameters typical for cardiac tissue: $r_{\text {ext }}=500 \Omega$ and $r_{\text {int }}=200 \Omega$ with the first time constant equal to $1.4 \mu \mathrm{s}$.

Mathematical modelling was performed for different cases beginning with the pure sine wave as errorless reference, the rectangular wave excitation and reference (Figs. 10 and 11), and continuing with different cases of simple approximations with sine waves (Figs. 12 and 13). 
(a)

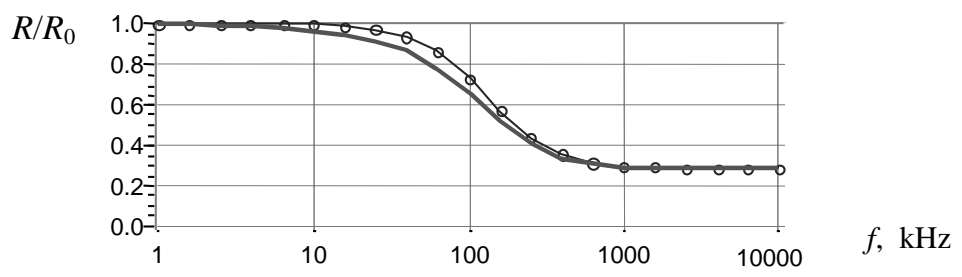

(b)

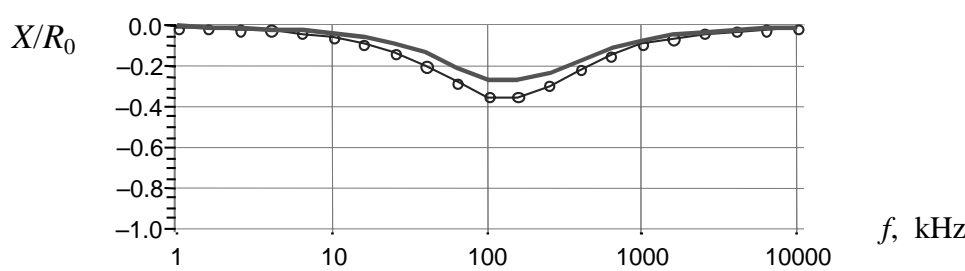

(c)

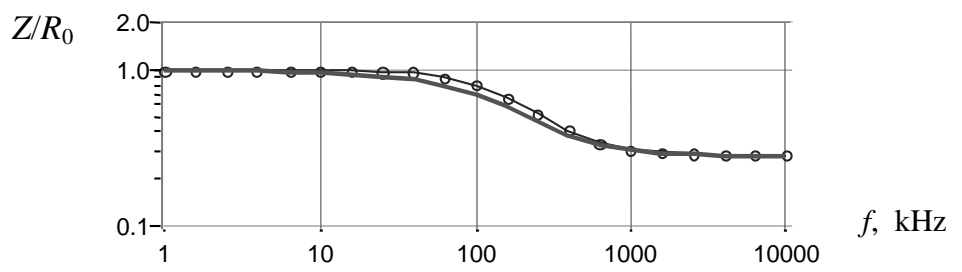

(d)

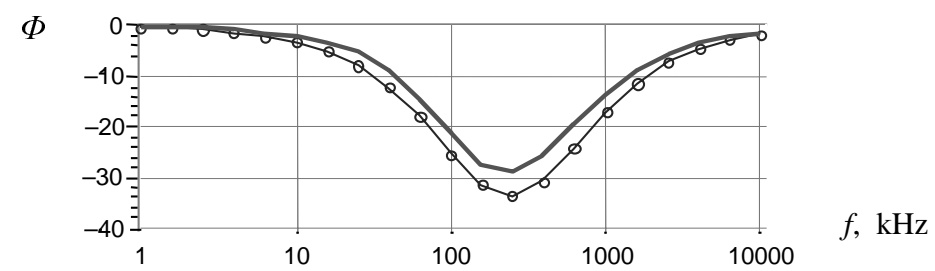

Fig. 10. The frequency response characteristics of the equivalent circuit determined using rectangular waveform of full duty cycle for both excitation and reference signals; curves without experimental points are the response in the case of pure sine waves. 
(a)

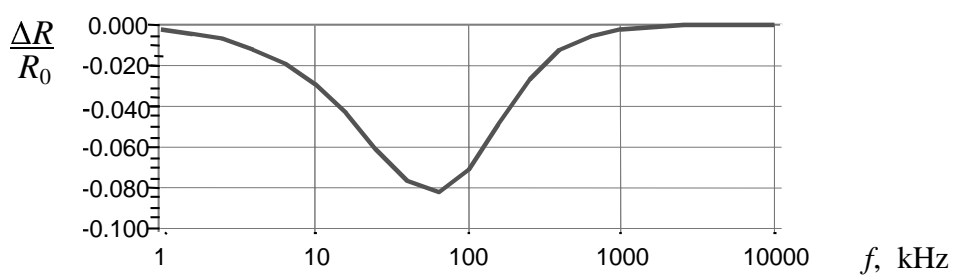

(b)

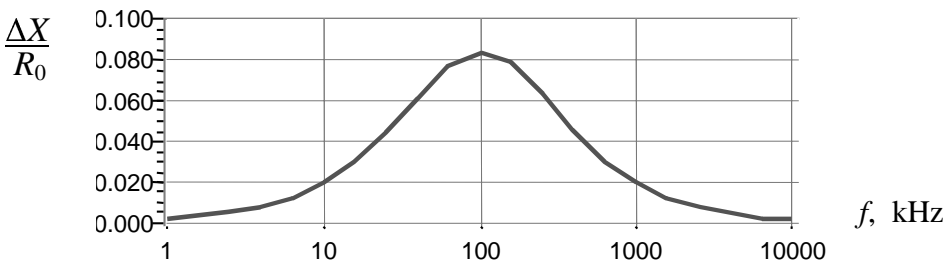

(c)

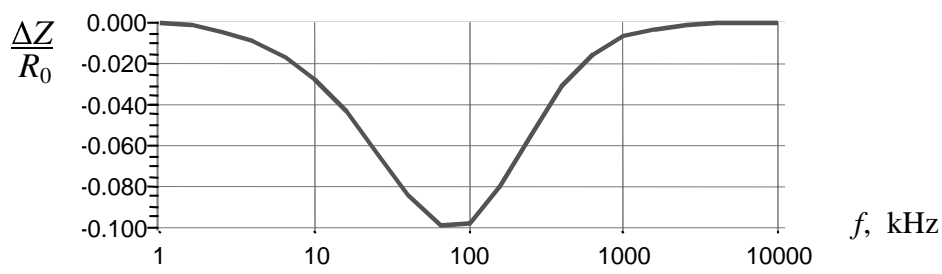

(d)

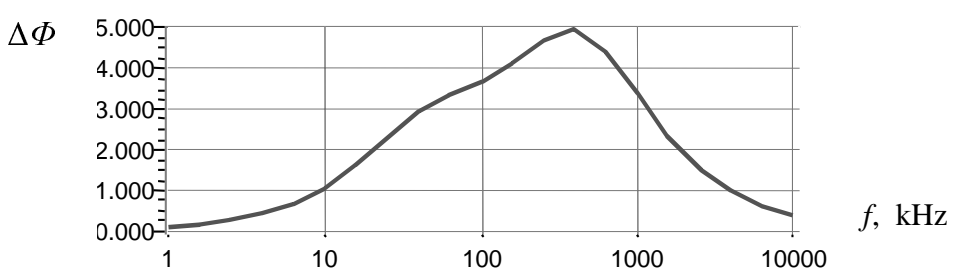

Fig. 11. Errors of determining the frequency response characteristics of the equivalent circuit in the case of using full duty cycle rectangular waveforms for both excitation and reference signals. 
(a)

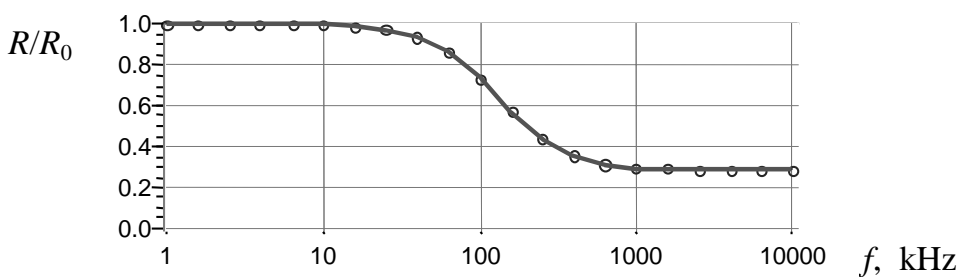

(b)

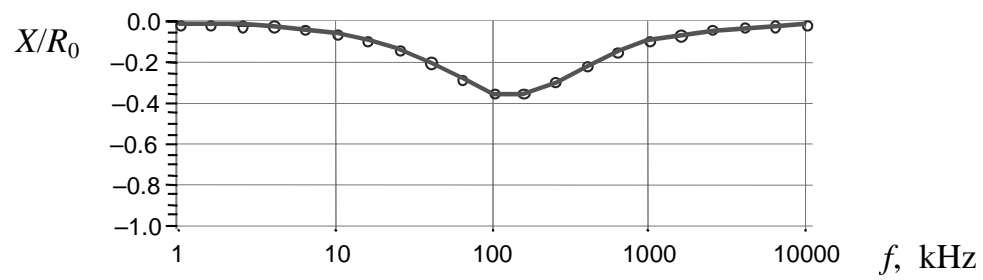

(c)

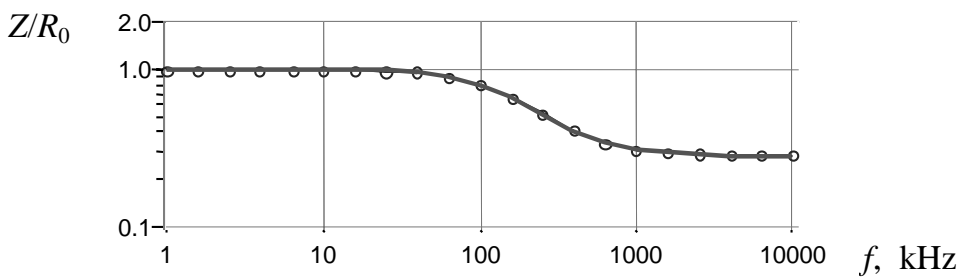

(d)

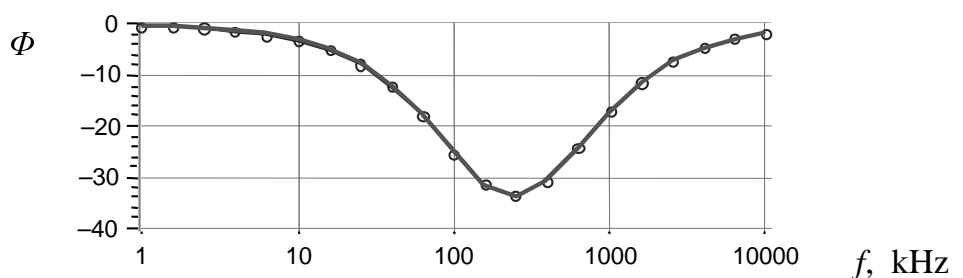

Fig. 12. The frequency response characteristics of the equivalent circuit determined using a rectangular waveform with narrowed duty cycle for both excitation and reference signals. The case of narrowing of the duty cycle by $18^{\circ}$ and $30^{\circ}$. 
(a)

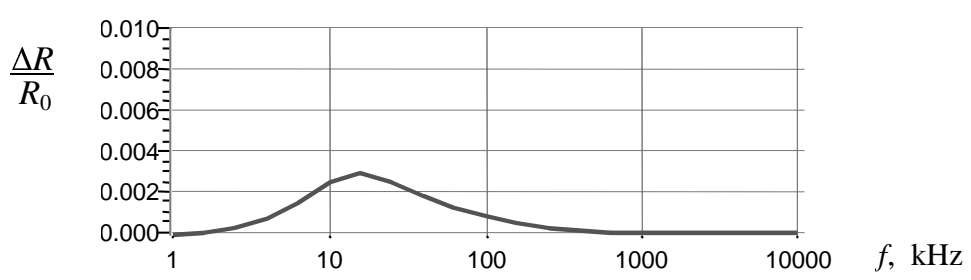

(b)

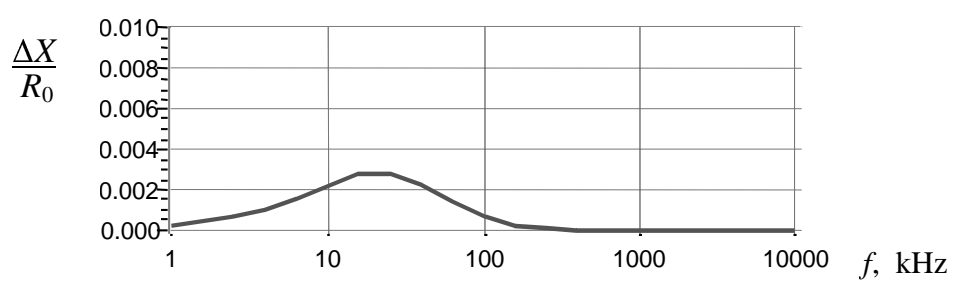

(c)

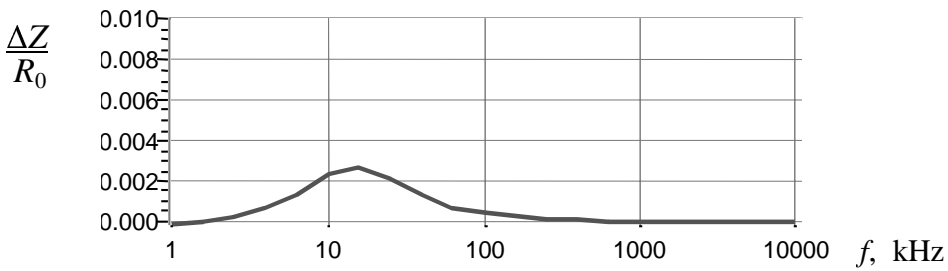

(d)

$\Delta \Phi$

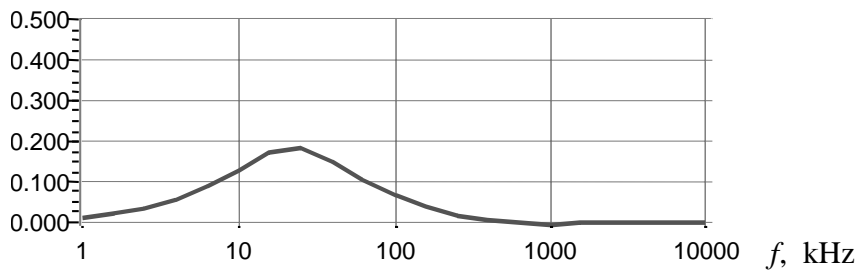

Fig. 13. Errors of determining the frequency response characteristics of the equivalent circuit in the case of using narrowed duty cycle rectangular waveform for both excitation and reference signals. The case of narrowing of the duty cycle by $18^{\circ}$ and $30^{\circ}$.

\section{CONCLUSIONS}

The solution of lock-in signal conversion on the basis of rectangular waveforms with narrowed pulse is simple enough to be used in the implantable and portable biomedical devices. In comparison with the case of using the full cycle rectangular waveforms, the magnitude error is decreased more than 10 times and the phase error is decreased up to 4 times. For the selected bioimpedance 
equivalent, this difference may reach even 30 times in favour of the case of narrowed by $18^{\circ}$ and $30^{\circ}$ pulses, for both magnitude and phase errors. Thus in spite of its simplicity, the method gives highly adequate phasor measurement results for the most cases of bioimpedance measurement and analysis.

\section{ACKNOWLEDGEMENT}

This work was partially supported by Estonian Science Foundation (grants Nos. 5892, 5897 and 5902).

\section{REFERENCES}

1. Webster, J. G. (ed.). Design of Cardiac Pacemakers. IEEE Press, Piscataway, NJ, 1995.

2. Meade, M. L. Lock-in Amplifiers: Principles and Applications. Peregrinus, London, 1983.

3. Grimnes, S. and Martinsen, Ø. G. Bioimpedance and Bioelectricity Basics. Academic Press, San Diego, 2000. (Chapter 6.3. Bridges, Impedance Analysers, Lock-in Amplifiers).

4. Min, M., Märtens, O., and Parve, T. Lock-in measurement of bio-impedance variations. Measurement, 2000, 27, 21-28.

5. Min, M. and Parve, T. Improvement of the vector analyser based on two-phase switching mode synchronous detection. Measurement, 1996, 19, 103-111.

6. Min, M., Parve, T., and Ronk, A. Design concepts of instruments for vector parameter identification. IEEE Trans. Instrum. Meas., 1992, 41, 50-53.

7. Min, M., Parve, T., Kukk, V., and Kuhlberg, A. An implantable analyzer of bio-impedance dynamics: mixed signal approach. IEEE Trans. Instrum. Meas., 2002, 51, 674-678.

8. Land, R. Piecewise linearly approximated sine wave for dynamic quality tests of A/D converters. Proc. Estonian Acad. Sci. Eng., 2000, 6, 113-119.

9. Min, M., Kink, A., Land, R., and Parve, T. Method and device for measurement of electrical bio-impedance. Patent application P200200677 (Estonia), Dec. 6, 2002, International Patent Application PCT/EE03/00006, filed Nov. 28, 2003.

10. Kuhlberg, A., Land, R., Min, M., and Parve, T. PWM based lock-in bioimpedance measurement unit for implantable medical devices. In Proc. XVII IMEKO World Congress "Metrology in the 3rd Millennium”, Dubrovnik, 2003, 1710-1713.

\section{Signaali sünkroonmuundamise täiustamine elektrilise bioimpedantsi mõõtmiseks}

\section{Toomas Parve ja Raul Land}

Esitatud on meetod elektrilise bioimpedantsi mõõturites signaali muundamiseks kasutatavate lülitirežiimsete sünkroonmuundite (lock-in converters) täiustamiseks, mis põhineb nelinurkse signaali poolperioodi impulsi lühendamisel. Lahendus on suunatud selliste faasivigade vähendamisele, mis on põhjustatud lülitirežiimsete sünkroonmuundite tundlikkusest sisendsignaali kõrgemate paaritute harmoonikute suhtes. Meetod on ette nähtud kasutamiseks in vivo mõõtmistel implanteeritavates ja portatiivsetes meditsiiniseadmetes. Täiustus on teostatav minimaalse skeemitehnilise keerukuse ja energiatarbe kasvuga. 